\title{
La dimension argumentative dans les textes poétiques : marques formelles et enjeux de lecture
}

Arguing in poetry: how it can be analyzsed and how it challenges our reading

\section{Michèle Monte}

\section{(2) OpenEdition \\ 1 Journals}

\section{Édition électronique}

URL : http://journals.openedition.org/aad/2511

DOI : 10.4000/aad. 2511

ISSN : 1565-8961

Éditeur

Université de Tel-Aviv

Référence électronique

Michèle Monte, "La dimension argumentative dans les textes poétiques : marques formelles et enjeux de lecture », Argumentation et Analyse du Discours [En ligne], 20 | 2018, mis en ligne le 15 avril 2018, consulté le 23 septembre 2019. URL : http://journals.openedition.org/aad/2511 ; DOI : 10.4000/aad. 2511

Ce document a été généré automatiquement le 23 septembre 2019.

\section{c) (†) $९$}

Argumentation \& analyse du discours est mis à disposition selon les termes de la licence Creative Commons Attribution - Pas d'Utilisation Commerciale - Pas de Modification 4.0 International. 


\title{
La dimension argumentative dans les textes poétiques : marques formelles et enjeux de lecture
}

Arguing in poetry: how it can be analyzsed and how it challenges our reading

\author{
Michèle Monte
}

\section{Cadre théorique}

1 Lorsque j'ai entrepris pour ma thèse d'étudier l'énonciation dans les poèmes de Philippe Jaccottet (Monte 2002), j'ai adopté une approche pragmatique qui s'est attachée entre autres à analyser le dialogisme inhérent à cette œuvre par une étude des négations, des interrogations, des connecteurs concessifs. J'ai voulu restituer à ces poèmes leur dimension de parole adressée et de débat, tout à la fois sur les conditions de la parole poétique et sur la façon de vivre (l'éthique) dans un monde traversé de forces contradictoires, et je l'ai fait en partant d'une étude des marqueurs indiquant des points de vue en confrontation. Dans des travaux ultérieurs, j'ai étudié les différentes manifestations de l'ethos en poésie, qu'il s'agisse du rapport à l'autre dans les discours représentés, des choix lexicaux, syntaxiques et rythmiques, ou de la construction du recueil (pour une synthèse, voir Monte 2016). Cet ethos est un des enjeux de la production/réception du poème, non pas parce qu'il contribuerait à convaincre le lecteur d'adhérer à une opinion explicite portée par un garant, mais parce qu'il lui propose une manière d'être et de dire qui est l'objet même du texte. L'enjeu de la parole poétique de type lyrique ${ }^{1}$ réside en effet principalement dans la transmission d'une expérience qui a ceci de spécifique qu'elle est inséparable d'un travail sur le matériau langagier. Certains poètes ont voulu théoriser cette spécificité en opposant par exemple, comme le fait Yves Bonnefoy, le langage de la poésie et celui du concept. D'autres ont refusé une dichotomie aussi radicale. Mais, dans tous les cas, la scène d'énonciation construite par le poème fait partie intégrante de son interprétation, aussi bien que la façon dont s'y opèrent la cohésion textuelle et la 
référenciation du monde. L'énonciation lyrique possède des caractéristiques propres (Monte 2003 et à paraitre) qui brouillent la différence entre situation spécifique et générique, entre émetteur et récepteur, cependant que le travail sur la dimension sensible du langage (rythme, sonorités) et sur sa dimension sémantique (en bouleversant souvent les représentations doxiques $\mathrm{du}$ monde) sollicitent la participation active du lecteur, amené de ce fait, lors du travail interprétatif, à vivre une expérience et pas simplement à construire une représentation du monde. C'est pourquoi le poème possède à certains égards une forte dimension argumentative, comme je vais m'employer à le montrer ici. Cependant la justification en est bien souvent absente, le poème s'attachant plutôt à construire une sorte d'évidence. La notion de dimension argumentative élaborée par Amossy (2012) est bien appropriée pour décrire ce type de relation avec le destinataire où c'est celui-ci qui, en s'incorporant le texte, en adopte les points de vue ${ }^{2}$. Néanmoins il arrive que certains poèmes possèdent une argumentativité beaucoup plus directe que le concept de dialogisme aide à appréhender.

2 Dans le débat qui oppose Amossy à d'autres chercheurs, je me propose donc, à propos du poème, de monter que l'argumentativité peut y être conçue de façon graduelle (Plantin 2016: 80). Il est certes légitime, ne serait-ce que pour aider les lecteurs novices à catégoriser les textes ou pour évaluer la cohérence d'un passage, de vouloir distinguer un noyau dur de textes argumentatifs possédant une visée de justification et impliquant une séquentialité spécifique due à la relation d'étayage (Micheli 2012). Or cette relation est bien souvent absente des textes poétiques, comme en sont absentes les concessions au sens strict du terme ${ }^{3}$. En revanche, la visée de positionnement que Micheli pose également comme définitoire de l'argumentation me parait présente dans tout texte poétique, et plus largement dans tout texte littéraire, en raison de la nature même du discours littéraire qui se construit par positionnements successifs à l'intérieur d'un champ bien étudié par Bourdieu (1992). Plus précisément, chaque texte littéraire, de par la réflexivité langagière qui le caractérise, montre son positionnement, même s'il ne le dit pas explicitement par des reprises de discours autres. C'est pourquoi je me propose ici de distinguer différents degrés d'argumentativité dans les textes poétiques: nous verrons au fil des exemples étudiés qu'argumenter en poésie peut porter sur le dit ou le dire, mais aussi que le positionnement adopté, conflictuel ou pas, est parfois très visible, parfois naturalisé par une énonciation qui efface le dialogisme inhérent à toute argumentation.

Précisons pour terminer la présentation de mon cadre théorique que j'adopte l'analyse énonciative de Rabatel qui distingue le locuteur primaire et l'énonciateur primaire: «le locuteur est l'instance qui profère un énoncé, dans ses dimensions phonétiques et phatiques ou scripturales, selon un repérage déictique ou indépendant d'ego, hic et nunc.» (Rabatel 2008: 7). Les énonciateurs sont les sources de points de vue (PDV) sur les objets de discours, et l'énonciateur premier E1 (en syncrétisme avec le locuteur premier L1) «subsume tous les contenus propositionnels qu'il assume» (ibid.). L1/E1 prend en charge ses propres PDV, et impute aux énonciateurs seconds des PDV qu'il prend en compte de façon neutre ou en manifestant son accord et son désaccord (Rabatel 2009). Un PDV est « un énoncé qui prédique des informations sur n'importe quel objet de discours, en donnant non seulement des renseignements sur l'objet (relatifs à sa dénotation), mais aussi sur la façon dont l'énonciateur envisage l'objet " (Rabatel 2017: 43). Un PDV peut ainsi correspondre à des propos rapportés, à des 
perceptions représentées, ou se manifester essentiellement par des choix de référenciation, comme nous le verrons.

\section{Conflits de points de vue}

Dans cette première partie, j'étudierai des poèmes où se manifestent plusieurs énonciateurs, voire locuteurs, exprimant des PDV contrastés plus ou moins étayés.

\section{Marqueurs explicites}

Certains poèmes sont organisés explicitement en séquences argumentatives : Adam (1990 : 227-236) montre brillamment comment la leçon profonde du poème de Queneau "Veille » résulte de l'enchainement des connecteurs «si... certes... mais ». Dans ces poèmes, L1/E1 débat avec lui-même ou avec d'autres énonciateurs, comme on peut le voir dans ces extraits de « Parler ", poème issu de Chants d'en bas de Jaccottet composé de huit parties numérotées :

(1)

\section{Section 1 :}

Parler est facile, et tracer des mots sur la page, en règle générale, est risquer peu de chose [...] et j'aurai beau répéter «sang » du haut en bas de la page, elle n'en sera pas tachée, ni moi blessé.

Aussi arrive-t-il qu'on prenne ce jeu en horreur [...]

Section 3 :

Parler pourtant est autre chose, quelquefois que se couvrir d'un bouclier d'air ou de paille... Quelquefois c'est [...]

Parler ainsi, ce qui eut nom chanter jadis et que l'on ose à peine maintenant, est-ce mensonge, illusion? [...]

Section 6 :

J'aurais voulu parler sans images, simplement pousser la porte...

Section 7 :

Parler donc est difficile, si c'est chercher... chercher quoi ? [...]

[Fin hors numérotation:]

(Je t'arracherais bien la langue, quelquefois,

sentencieux phraseur. Mais regarde-toi donc [...])

(Chants d'en bas, 41-51)

Les connecteurs logiques (" pourtant », « aussi », « donc »), l'emploi d'« avoir beau » et du marqueur argumentatif «bien», les lexèmes évaluatifs («facile», "prenne en horreur ", "mensonge, illusion ", « difficile ») qui portent tous sur le même objet de discours, à savoir la parole, permettent de discerner des positions opposées sur l'écriture poétique : pour le PDV (A), elle n'est qu'une échappatoire mensongère alors que le PDV (B) croit possible une parole juste. Comme on peut le voir dans les extraits des sections 1 et 3, ces PDV sont étayés par une description tantôt flatteuse, tantôt dépréciative de l'activité de parole. L'enjeu du poème se dégage peu à peu : il s'agit, en dépassant cette opposition des PDV, de déterminer les conditions qui permettront à la parole de tenir face à la mort qui « f[ai]t pourrir /même les mots » (ibid., 47). La section 
7 examine ces conditions. Elle est toutefois suivie d'une section qui congédie la parole au profit de l'action : " habille-toi d'une fourrure de soleil et sors ", en mettant en scène un autre énonciateur qui traite le poète de " faux mendiant ", de " coureur de linceuls " et qui est porteur d'un point de vue (C) surplombant. C'est à lui que l'on attribue également le poème en italiques non numéroté qui clôt « Parler ». On pourra également analyser à la lumière de ces positionnements le passage d'une alternance "on/nous ", « je », « vous » à une alternance « je » vs « tu » quand surgit le point de vue (C). Dans la première partie, le «je » a une valeur quasi générique, de même que le "vous » et le "on»: les référents des trois pronoms ne se distinguent pas par des positions différentes, tous épousent tour à tour (A) et (B). Dans la deuxième partie, le « je » blâme le «tu» et apparait comme la conscience critique du locuteur. On passe ainsi d'un dialogisme interlocutif où le locuteur se fait le porte-parole d'une communauté en quête de vérité à un dialogisme intralocutif (Bres et al. 2016) plus conflictuel où le « je » cherche à disqualifier le « tu » par des arguments ad hominem.

7 L'exemple suivant offre également des marqueurs d'argumentation mais, contrairement à (1), les thèses en présence ne sont pas explicitées :

(2)

Trente-quatre lances enchevêtrées peuvent-elles composer un être? Oui, un Meidosem. Un Meidosem souffrant, un Meidosem qui ne sait plus où se mettre, qui ne sait plus comment se tenir, comment faire face, qui ne sait plus être qu'un Meidosem.

Ils ont détruit son « un ».

Mais il n'est pas encore battu. Les lances qui doivent lui servir utilement contre tant

d'ennemis, il se les est passées d'abord à travers le corps.

Mais il n'est pas encore battu.

(Henri Michaux, « Portrait des Meidosems » 1949, fragment 5, p.202)

«Portrait des Meidosems» est une des quatre parties de La vie dans les plis: le poème comprend soixante-neuf fragments non numérotés séparés par des étoiles. Comme l'indique son titre, il ne s'agit pas a priori d'un texte argumentatif, mais du portrait de créatures imaginaires, comme dans Ailleurs. Néanmoins les fragments se caractérisent très souvent par des formes de dialogisme. On relève ici une interrogation suivie d'une réponse confirmative, puis d'une phrase nominale dont les relatives prédicatives suggèrent des inférences qui sont ensuite contestées par l'énoncé « il n'est pas encore battu» précédé de "mais». On peut résumer le texte sous la forme: "Certes ce Meidosem est en piteux état mais il n'est pas encore battu». Le résumé montre que ce qui manque, c'est la conclusion qu'on pourrait tirer de ce constat. On peut cependant l'inférer et la formuler ainsi : "Une victoire du Meidosem est encore possible». On observe également que L1/E1, dépourvu de marques déictiques, est fortement présent comme sujet modal : après une interrogation qui anticipe sur le scepticisme du lecteur, il oriente la présentation des faits de façon à nous faire inférer la conclusion favorable au Meidosem. Une inversion des énoncés qui se trouvent à gauche et à droite des deux «mais» conduirait à une inférence inverse ${ }^{4}$. Le fragment est ainsi doté d'une dimension argumentative indiscutable. Cependant l'étayage de la conclusion repose surtout sur la force de conviction du locuteur, manifestée par la répétition de « Mais il n'est pas encore battu. » Le fragment présente ainsi le paradoxe d'une allure logique masquant une fragilité intrinsèque. Ceci me parait caractéristique de l'humour de Michaux qui se plait à dérouter ses lecteurs en leur proposant des textes dont la structure superficielle pousse à chercher des raisons de croire à ce qu'il nous raconte alors que le raisonnement est en réalité lacunaire. 
«Portrait des Meidosems » présente toutes les caractéristiques d'un texte dialogique :

- dislocations gauche et droite exhibant le choix de l'objet de discours : «Ce troupeau qui vient là, comme des pachydermes lents, avançant à la file, leur masse est et n'est pas» (203)

- énoncés négatifs inscrivant en creux la position combattue par l'énonciateur de nég-p

- interrogations sur le sens à donner à un évènement: «Un ciel de cuivre le couvre. Une ville de sucre lui rit. Que va-t-il faire?» (207)

-37 occurrences de « mais », quatre de "pourtant » et « cependant »

- emploi de « oui » et «non » à plusieurs reprises.

10 S'il s'agissait simplement de nous faire éprouver de l'empathie pour ces êtres étranges et de nous pousser à souhaiter sinon leur victoire, du moins leur préservation, l'appui sur les émotions et les ressources mobilisées d'ordinaire par l'épidictique (lexèmes affectifs, figures d'amplification, travail sur le rythme, voir Koren 2004) - d'ailleurs présentes dans ce texte - suffiraient. En construisant deux PDV, un de soutien aux Meidosems, l'autre, d'indifférence, de non-participation à leurs efforts, le poème introduit une perplexité chez le lecteur, une distance critique, comme on peut l'observer à nouveau dans le fragment 39 :

(3)

Le voici le nœud indivisible et c'est un Meidosem. Tout éruption, si on l'écoutait, mais c'est un nœud indivisible.

Profondément, inextricablement noué. Sa jambe cessant d'être jambe si jamais elle l'a été, balai terminal d'une poitrine serrée qui elle aussi montre la corde et le jute. Quel étranglé ne parle un jour de se libérer? Les tables elles-mêmes parlent, à ce qu'on dit, de se libérer de leurs fibres.

(Michaux $2001: 213$ )

11 Dans ce fragment, on peut repérer des procédés discursifs qui rendent le Meidosem présent et proche - déictique "le voici », insistance créée par les deux adverbes du début du $\S 2$, description pathétique de son corps -, mais on observe aussi des éléments de mise à distance. Le PDV de L1/E1, qui, en (2), soutenait les Meidosems s'oppose ici à celui du Meidosem : là où le Meidosem se présente comme un être en éruption, L1/E1 voit surtout un être noué, et il nous amène, sans s'engager lui-même directement, à considérer comme irréaliste son aspiration à se libérer ${ }^{5}$.

12 Du point de vue générique, «Portrait des Meidosems » se caractérise ainsi par son hybridité : il conjoint les propriétés de la description empathique et de l'examen critique. L1/E1 tient en bride l'émotion et il le fait en amenant l'allocutaire à s'interroger sur la nature des Meidosems, sur leurs chances de survie, sur la rationalité ou la bizarrerie de leurs comportements. De là un intense dialogisme. Mais ce poème nous permet d'établir une distinction importante entre degré de dialogisme et degré d'argumentativité. En effet le dialogisme, qui est ici surtout de nature interlocutive, anticipe sur les erreurs d'interprétation du lecteur, le sollicite par des questions, lui impute des points de vue à réfuter, mais il ne conduit pas nécessairement à une argumentation complète. Dans la perspective de Micheli, on peut dire qu'il satisfait à la visée de positionnement, mais pas à celle d'étayage.

\section{Dialogisme interdiscursif et discours rapportés}

13 Dans les textes que je vais examiner à présent, L1/E1 problématise des PDV qu'il ne se borne pas à prendre en compte dans ses propres énoncés, mais dont il reprend, à des 
fins polémiques, les formulations mêmes, telles qu'elles ont pu être cristallisées dans l'interdiscours. Contrairement à ce qu'a pu écrire Bakhtine (1978), tous les poèmes ne sont pas portés par une voix homogène. Certains recueils sont au contraire tissés de références à des discours multiples et leur interprétation dépend crucialement du rôle que, dans l'économie de l'œuvre, on pourra attribuer à ces discours représentés ${ }^{6}$. Voyons par exemple ce poème extrait de Leçons de Jaccottet :

(4)

On le déchire, on l'arrache,

cette chambre où nous nous serrons est déchirée,

notre fibre crie.

Si c'était le « voile du Temps » qui se déchire,

la « cage du corps » qui se brise,

si c'était l'« autre naissance »?

On passerait par le chas de la plaie,

on entrerait vivant dans l'éternel...

Accoucheuses si calmes, si sévères,

avez-vous entendu le cri

d'une nouvelle vie?

Moi je n'ai vu que cire qui perdait sa flamme

et pas la place entre ces lèvres sèches

pour l'envol d'aucun oiseau.

(Leçons, Poésie/Gallimard, 25)

Dans ce poème, les guillemets isolent des expressions qui renvoient à des discours sur la mort que L1/E1 prend tout d'abord en compte à titre d'hypothèse par les formes en «si + IMP - conditionnel » puis qu'il questionne en interrogeant les " accoucheuses " avant de les contester par la négation exceptive et le pronom d'insistance qui oppose l'expérience $\mathrm{du}$ « je » aux propos consolateurs. La mise en scène des discours rapportés est porteuse d'une forte argumentativité, puisqu'elle oppose non seulement deux opinions mais aussi deux séries de formulations dont l'hétérogénéité est marquée : aux périphrases platoniciennes s'oppose la sécheresse de la dernière laisse ${ }^{8}$. Ce dialogisme interdiscursif est ici marqué mais ponctuel. Il peut parfois être systématisé et devenir la raison d'être du livre, comme nous allons le voir avec cet extrait d'Un $A B C$ de la barbarie de Jacques-Henri Michot :

(5)

Fête du travail: les syndicats défilent en ordre dispersé de la Bastille à la

République. (vieux)

Feux aux poudres, feux de l'actualité, feux des projecteurs

Feux roulants des questions

Feux verts $C f$. Satisfaction dans les capitales européennes

Feuilletons politico-médiatiques de l'été

Fichages

Fidélisation de la clientèle

(Vieux) Fiefs électoraux

(72-73, les caractères gras sont de l'auteur)

Un $A B C$ de la barbarie se présente comme une liste par ordre alphabétique de mots, syntagmes et énoncés empruntés à la phraséologie journalistique, économique et/ou politique du moment, entrecoupés par des citations d'écrivains en italiques, des titres d'œuvres musicales et des reproductions de tableaux qui en apparaissent dès lors comme le contrepoint et qui sont, elles aussi, rangées par ordre alphabétique des noms d'auteur. Ces citations sont commentées par d'abondantes notes de bas de page qui sont attribuées par la préface à un certain François B, lequel évoque le rapport 
qu'entretenaient avec ces écrivains et artistes ses amis Barnabé et Jérémie, respectivement auteur et éditeur du livre, décédés. Voici un exemple de ces notes, après une citation de Malachie :

(6)

$\mathrm{N}^{\circ}$ 3003. Jamais je n'oublierai la troublante déclaration que Barnabé nous fit, à Jérémie et à moi-même, une nuit de son dernier hiver, vers 3 heures du matin : «Malachie, comme vous le savez l'un et l'autre, n'est pas un garçon que ses père et mère auraient appelé Malachie. Nul prénom qui lui soit propre, mais la seule désignation d'une fonction: maléaki, le messager. Eh bien, très chers, je me sens assez proche de ce Malachie-là, qui, en somme, n'existe pas » (122, italiques de l'auteur).

La dimension argumentative de ce livre réside tout entière dans son organisation : il ne dénonce pas explicitement le prêt-à-penser des dominants mais il en fait entendre la vacuité ou le caractère oppressif par la juxtaposition interne (la succession des emprunts à la doxa médiatique) et externe (le contrepoint des citations et références aux œuvres d'art) et par les notes qui construisent progressivement le portrait d'une amitié intellectuelle entre trois personnages de " résistants » à l'ordre dominant à qui il arrive d'évaluer explicitement les discours cités.

Dans les exemples (1) à (6), le locuteur anticipe sur des questions du lecteur, lui impute des attentes ou des inférences, engage le débat avec lui, conteste explicitement certains PDV, cite certains discours pour les critiquer et d'autres pour les proposer à notre admiration. Toutes les formes du dialogisme sont présentes et aisément repérables, et l'on voit que la poésie peut très bien mettre en débat des façons de dire et de penser, notamment par la construction des recueils où les textes se répondent les uns aux autres. Il arrive cependant que l'imbrication des PDV, comme dans les exemples (2) et (3), soit assez subtile pour brouiller les cartes. Cette complexité s'accroit, nous allons le voir, quand ce qui est mis en débat n'est pas un contenu de pensée, une façon de dire ou un comportement, mais la pratique littéraire elle-même.

\section{Le travestissement et le pastiche : argumenter en prenant la littérature pour objet}

Au sein des pratiques hypertextuelles (Genette 1982), les parties en romains d'Un ABC de la barbarie constituent un bon exemple de parodie. Celle-ci n'imite pas, elle reprend et détourne un hypotexte précis : toutes les expressions empruntées par Michot et citées en (5) pourraient être retrouvées en $\mathrm{x}$ exemplaires dans les médias. D'autres pratiques dialoguent avec des textes sources en s'en inspirant mais sans les reprendre tels quels : il s'agit du travestissement et du pastiche ${ }^{9}$, qui se distinguent aussi bien par leur structure que par leur objectif. Le travestissement burlesque «modifie le style sans changer le sujet » (Genette 1982: 35) : il consiste dans sa forme la plus basique à mettre des propos triviaux dans la bouche de personnages nobles. Un bon exemple en serait la chute du sonnet «Le Cid» de Georges Fourest : Chimène s'exclame en effet, en voyant passer Rodrigue, «Qu'il est joli garçon, l'assassin de papa!». Dans La Négresse blonde (1913), Fourest évoque ainsi plusieurs tragédies classiques en mettant dans la bouche des héros force anachronismes et expressions familières ou vulgaires ${ }^{10}$. On peut défendre une conception plus large du travestissement en considérant qu'il concerne 
également la reprise dans un style familier des topiques récurrentes de la poésie. Un exemple nous en est fourni par le célèbre poème de Rimbaud « Ma bohème " ${ }^{11}$ :

(7)

Ma bohème (Fantaisie)

Je m'en allais, les poings dans mes poches crevées ;

Mon paletot aussi devenait idéal ;

J'allais sous le ciel, Muse ! et j'étais ton féal ;

Oh ! là ! là ! que d'amours splendides j'ai rêvées !

Mon unique culotte avait un large trou.

- Petit-Poucet rêveur, j'égrenais dans ma course

Des rimes. Mon auberge était à la Grande-Ourse.

- Mes étoiles au ciel avaient un doux frou-frou.

Et je les écoutais, assis au bord des routes,

Ces bons soirs de septembre où je sentais des gouttes

De rosée à mon front, comme un vin de vigueur ;

Où, rimant au milieu des ombres fantastiques,

Comme des lyres, je tirais les élastiques

De mes souliers blessés, un pied près de mon cœur !

Le texte doit être lu comme une critique des évocations topiques du poète inspiré. Il mêle volontairement les topoï romantiques (la fidélité à la Muse, les promenades nocturnes sous les étoiles, l'union avec la nature, le consentement à la pauvreté par désintéressement) et le choix d'un lexique familier ou trivial ("poches crevées», " paletot », « Oh ! là ! là ! ", « culotte »12, « élastiques » comparés aux « lyres », « souliers blessés ») dont le lecteur d'aujourd'hui, habitué aux contrastes de tons, ne perçoit qu'imparfaitement la violence. Ce choc des registres est résumé dans le dernier hémistiche "un pied près de mon cœur ». Une telle pratique hypertextuelle a une finalité argumentative claire : il s'agit d'affirmer un positionnement anticonformiste, qui appelle un chat un chat et prétend renouveler la poésie, même si le locuteur mis en scène par l'énonciateur textuel ne renie qu'en partie les topoï et assume la part d'idéal de ses vagabondages. Le jeu entre le dire et le montrer est subtil : le poème respecte les règles du sonnet régulier et L1/E1 affirme son contentement de façon apparemment non dialogique. Seule une connaissance du contexte littéraire aide l'interprète à percevoir l'ironie et à poser un dialogisme entre L1/E1 et l'énonciateur textuel (l'auteur impliqué) $)^{13}$.

Le pastiche littéraire, quant à lui, est « une pratique mimétique visant à produire un texte (T2) en reprenant les traits stylistiques marquants d'un modèle (T1)» (Aron 2009 \$1). Ce modèle peut être le style d'un écrivain précis ${ }^{14}$ ou les traits d'un genre. L'énonciateur textuel signale généralement sa présence dans le paratexte, et, comme le dit Aron à propos du premier pastiche dont nous disposions, l'imitation par Socrate, dans le Ménexène de Platon, d'une oraison funèbre prononcée par Aspasie: "[1]a fonction du discours-cadre est de donner un code de lecture du discours encadré, de signaler le nom ou la nature du texte pastiché. Ce dispositif ne conduit cependant pas à disqualifier les propos attribués à Aspasie, mais à attirer l'attention de l'auditeur sur leur construction et leurs effets » (§ 2).

L'objectif du pastiche est en effet, depuis le $19^{e}$ siècle qui a vu l'essor de la pratique, de prouver la virtuosité de son auteur, mais aussi d'amener le lecteur à s'interroger sur les ingrédients d'un style, et plus généralement à prendre ses distances vis-à-vis de la doxa de l'auteur génial, inimitable. Cuvre reposant sur la connivence de lecteurs cultivés, le 
pastiche à la fois célèbre et critique la littérature. Examinons à cet effet un pastiche de Baudelaire écrit par Henri de Régnier et publié par La Revue de Paris le 15 avril $1921^{15}$ :

(8)

«Stances baudelairiennes"

Je veux chanter tout bas, ô beauté taciturne,

Le silence divin de tes beaux yeux fermés

En choisissant, parmi notre passé nocturne,

Les instants que ma vie aura le mieux aimés.

Sera-ce ce doux soir où, dans l'air qu'elle embaume,

Je me revois assis en de nobles jardins,

Respirant près de toi le magnétique arome

D'une fleur parfumée à l'odeur de tes mains?

Ou cet autre où, couchée au divan de paresse,

Dans le trouble désir d'un délice inconnu,

D'une incertaine, vague et furtive caresse

J'effleurai doucement l'ongle de ton pied nu?

Mais non! C'est cette nuit ardente et généreuse

Où, sans peur, sans remords, sans honte et sans aveux,

$\mathrm{Tu}$ laissas se poser ma lèvre aventureuse

Sur les trésors secrets de ton corps ténébreux.

Car cet obscur baiser, ô reine taciturne

De l'ombre favorable et des instants aimés,

A scellé notre pacte amoureux et nocturne

Mieux que ton cher silence et tes beaux yeux fermés!

Conformément aux règles qui gouvernent les pastiches les plus exigeants, ce texte reprend des thèmes, du vocabulaire, des structures énonciatives (apostrophes), syntaxiques (série d'adjectifs antéposés, circonstants antéposés) et rythmiques (enjambements, préposition "parmi » aux $7^{\mathrm{e}}$ et $8^{\mathrm{e}}$ syllabes) typiques des Fleurs du mal, tout en ne comportant aucun vers figurant dans le recueil. Henri de Régnier brasse le matériau verbal du recueil et compose un poème original mais typiquement baudelairien. L'argumentativité revêt ici une dimension métatextuelle et elle est indissociable de la scène d'énonciation : l'énonciateur textuel est censé être Baudelaire, mais le titre indique la supercherie et l'on a une dissociation entre le locuteur qui reproduit l'ethos de l'énonciateur Baudelaire et l'énonciateur textuel, le poète Henri de Régnier. Le poème apparait à la fois comme un hommage et comme un tour de force qui illustre son auteur. Régnier est un habitué du pastiche et on peut penser que ses pastiches argumentent en faveur d'une pratique de la littérature entendue comme une lecture assidue des grands auteurs et une capacité à trouver sa voie en sachant imiter les autres.

Le point commun, sur le plan linguistique, de toutes les pratiques étudiées ci-dessus, c'est qu'elles peuvent toutes être définies, selon les propositions de Bres, Nowakowska, Sarale (2016), comme des représentations dans un énoncé (E) soit d'un autre énoncé (e) plus ou moins reconnaissable selon qu'on a affaire à une citation (ex. 4 et 5), à une allusion ("Ma bohème ») ou à une reformulation («Stances baudelairiennes»), soit d'un PDV qui peut se trouver intégré à (E) - cas de la négation - ou être implicite mais reconstituable à partir de (E) qui nécessite pour être compris de poser un PDV opposé à celui qui est présent en (E) : les exemples (1), (2) et (3) combinent intégration et ellipse ${ }^{16}$ du PDV opposé. Nous allons passer à présent à un mode beaucoup plus oblique d'argumentation en basculant vers des poèmes qui proposent au lecteur des schématisations à visée épidictique. 


\section{L'argumentation épidictique}

24 sous deux (ou plusieurs) faces ou lorsqu'on indique que des discours bien différents peuvent en rendre compte, elle devient problématique. Le dialogisme a une vertu problématisante. Il s'oppose à l'évidence que Doury considère comme le but de l'épidictique: "L'épidictique, se donnant comme hors-débat, revendique une forme d'évidence. À cette évidence sont associés différents procédés discursifs qui relèvent non de l'intellection, mais de la mise en scène : il s'agit de conférer à ce dont on parle une forme de présence susceptible d'agir directement sur la sensibilité de l'auditoire " (2010: § 8). La place de l'épidictique dans l'argumentation a toujours été discutée, précisément en raison de l'effacement de la conflictualité qui en est constitutif. Alors que Plantin défend l'idée qu'« une situation langagière donnée commence à devenir argumentative lorsque s'y manifeste une opposition de discours » $(2016: 80)$, je poserai au contraire qu'un texte épidictique ${ }^{17}$, dans la mesure où il valorise ou dévalorise un objet de discours, est argumentatif. Mais la neutralisation du conflit ne signifie pas que L1/E1 se départit d'un certain jugement sur la réalité. Simplement celui-ci passe par d'autres moyens, et notamment par la schématisation ${ }^{18}$, au sens que Grize donne à ce mot : «Une schématisation a pour rôle de faire voir quelque chose à quelqu'un, plus précisément, c'est une représentation discursive orientée vers un destinataire de ce que son auteur conçoit ou imagine d'une certaine réalité » (1997 : 50).

\section{$(2004: 30)$ :}

(9)

Que la joie est simple au bout du cheminement obscur!

Comme ces minces pellicules donnent corps à la lumière !

Regarde comme il fond ce peu

de blanc tombé au fond de l'œil!

Les amandiers dans la nuit!

ô les dents de clarté !

Pulsation sourde d'étoiles

dans l'épaisseur de la terre -

Du point de vue illocutoire, le poème vise d'abord à faire partager une émotion : il enchaine une série d'actes expressifs, marqués par l'utilisation d'adverbes exclamatifs, par le «ô " lyrique et par une invitation adressée à l'allocutaire afin qu'il partage l'admiration et la joie du locuteur. Que l'on adopte ou non la théorie des actes de langage de Kissine et Dominicy ${ }^{19}$, on peut observer que L1/E1 ne fournit pas de raison de croire à ce qu'il dit: dans les deux premières laisses, les démonstratifs à valeur déictique créent un espace partagé. Ceci coïncide avec le recours à des structures phrastiques qui présentent les contenus des énoncés comme des évidences présupposées $^{20}$, communes à tous les membres de la collectivité. Il n'y a donc pas

Argumentation et Analyse du Discours, 20 | 2018 
d'assertion mais un rappel de choses connues fondant une communion expressive. Dans la troisième laisse, l'énonciation devient générique : il est question des amandiers en fleurs en général et pas seulement de ceux que L1/E1 a sous les yeux. Mais l'intensité expressive se poursuit encore sur deux vers, et L1/E1 s'appuie sur elle pour communiquer in fine sans l'argumenter une croyance exprimée par deux métaphores : les amandiers sont décrits comme les dents de la nuit personnifiée puis comme des étoiles surgies de la terre ${ }^{21}$. Si l'on s'interroge à présent sur la valeur perlocutoire de ce fragment, il me semble possible d'affirmer que l'énonciateur textuel veut, au moyen des structures exclamatives et de l'amplification cosmique de la dernière laisse, nous amener non seulement à croire mais même à éprouver, en convoquant des représentations supposées partagées, que les amandiers sont une source de joie et d'émerveillement en raison de leur clarté qui naît de la nuit et transcende donc les oppositions lumière/obscurité. Ce dépassement des contraires est un des topoï de la poésie (Monte 2008) et, dans une culture occidentale fondée sur le principe de noncontradiction, invite à une autre vision du monde et de la vie, ce qui ressortit à la dimension argumentative des textes, comme en relève également dans ce fragment l'invitation indirecte à la joie. Cependant ce contenu exhortatif ne se situe pas au niveau illocutoire et n'est pas étayé par des raisons, il repose sur une communion dans l'émotion. J'adopte ici l'analyse de Franken et Dominicy (2001) qui écrivent: "Nous supposerons ici qu'au contraire de ce qui se passe dans le délibératif, l'orateur de l'épidictique, cherchant à fournir un conseil "parénétique ", agit sur son auditoire en l'amenant à un certain état psychologique, sans que cet auditoire prenne conscience d'autre chose que de la nature expressive du discours produit » $(2001: 106)$.

Franken et Dominicy entendent par conseil "parénétique » un conseil «qui touche à des matières non controversées, provoquant ainsi une décision spontanée et évidente la proairesis » (ibid.: 104). La proairesis ou préférence éthique conduit à la praxis et s'appuie non sur un raisonnement mais sur l'adhésion à des valeurs communes réactivées par l'amplification. Comme l'épidictique, le poétique suscite une proairesis en " amenant l'auditeur à un certain état psychologique». On peut en revanche opposer l'univocité des discours épidictiques politiques ou religieux, au flou conceptuel des représentations évoquées par le poème, notamment en raison de la présence fréquente de paradoxes cognitifs (Michaux et Dominicy 2001). Ce flou conceptuel est toutefois compensé en (7) par l'intensité émotionnelle. L'exemple suivant (Grosjean 1996: 67), représentatif de ce que Maulpoix a appelé le lyrisme critique (2009), va nous confronter à une variété paradoxale d'épidictique où l'émotion semble tenue à distance :

(10)

Bol de thé

Le rosier rouge en fleur contre la grange.

Longueur du jour puisque l'été commence.

Déjà les blés sur les coteaux jaunissent.

Un pavot noir s'ouvre au bord des moissons.

Un merle chante au milieu d'un buisson.

L'ombre de la maison nous est propice.

L'échelle appuie son ombre au mur qui penche.

Le soleil ne descend que par degrés.

Le ciel repose au fond d'un bol de thé.

L'ombre effilée d'une herbe sous ma manche.

On peut remarquer que ce poème contient quelques évaluatifs («longueur ", « déjà », «ne...que ») qui situent certains des phénomènes évoqués sur une échelle quantitative, 
toujours en rapport avec la localisation de l'instant dans l'année. On observe aussi un adjectif faiblement euphorique au vers 6 ("propice») associé au "nous». En fonction de stéréotypes culturels partagés (sur l'agrément procuré par les fleurs, le chant des oiseaux, le thé), on peut en conclure que le moment décrit est vécu comme agréable par le locuteur qui apparaît au dernier vers. Cependant rien n'est dit explicitement, et l'enjeu du texte est ailleurs. Comme dans les haïkus, il s'agit de poser un certain nombre de rapports entre des éléments d'un même paysage : contrastes de couleurs, de lumière, de positions dans l'espace (loin $v s$ près, vertical $v s$ horizontal), d'expériences perceptives (immensité du ciel vs petitesse du bol, mise en regard de l'échelle et de l'herbe, du pavot et du merle). Le texte possède une dimension picturale et doit être lu comme une invitation à la contemplation. La régularité des décasyllabes (tous césurés 6-4 ou 4-6) est en accord avec l'impression paisible qui se dégage des énoncés. La présence de rimes, plutôt rares dans ce livre, associe fortement les énoncés homéotéleutes, renforçant ainsi les parallélismes et les contrastes sémantiques. On peut qualifier ce texte de descriptif, et donc considérer qu'il ne possède pas de dimension argumentative. Cependant les travaux sur la description, depuis Barthes (1968) et Hamon (1994), ont bien montré que, dans le roman, celle-ci est au service d'une visée englobante, qu'il s'agisse d'ancrer la narration dans le réel, de fournir des indices d'une évolution du récit, d'indiquer, par les subjectivèmes, l'état d'esprit du locuteur principal ou d'un personnage: Adam et Petitjean (1989: 47) parlent de la « sursignifiance » des descriptions dans un récit.

En poésie, la description ou ekphrasis est un des lieux du texte où se manifeste le pouvoir du poète de "faire voir» et de rivaliser avec la nature. Elle se déploie notamment dans l'évocation du locus amoenus : il s'agit de recréer par les mots un lieu idyllique et de prendre ainsi en charge une nostalgie du paradis perdu qui est un thème récurrent de la tradition poétique. On peut ainsi en conclure comme Jean Lebel (2002) qu'à l'origine, "la description n'est jamais neutre, se fait éloge ou blâme et porte toujours la marque de la subjectivité du descripteur». À l'aune de cet ancrage historique, la description neutre du «Bol de thé » affirme à la fois une continuité et une différence : d'une part, elle indique que la poésie fait toujours sienne l'ambition de recréer par les mots un lieu et les sensations qui y sont attachées, d'autre part, elle revendique le refus de l'emphase, la simplicité et l'effacement de l'énonciateur ${ }^{22}$. Ces trois traits correspondent à la modestie des poètes contemporains, à leur posture critique qui se méfie d'un langage qui ferait illusion et empêcherait l'accès à la vérité, et à leur volonté de laisser la première place au réel en disparaissant au profit de ce dont ils parlent. Dès lors, l'écriture de «Bol de thé » se fait argumentative par son refus même de l'argumentation, et si elle défend un certain rapport au réel, c'est uniquement en l'incarnant dans l'écriture même du texte. Dans ses textes réflexifs, Jaccottet s'est exprimé parfois sur cette quête du simple, et le passage ci-dessous de La Promenade sous les arbres me semble en consonance avec le poème de Grosjean :

(11)

Mais vient un moment où l'on s'aperçoit que les images même les moins fausses gênent encore notre souci de la vérité absolue, et qu'il faudrait les dépasser. [...] Le rêve qui nous saisit à ce moment-là est celui d'une transparence absolue du poème, dans lequel les choses seraient simplement situées, mises en ordre, avec les tensions que créent les distances, les accents particuliers que donne l'éclairage, la sérénité aussi que suscite une diction régulière, un discours dépouillé de tout souci de convaincre l'auditeur, de faire briller celui qui discourt, ou, à plus forte raison, de lui 
valoir une victoire de quelque espèce que ce soit.

(Jaccottet $1988: 119-120$, je souligne)

31 On voit ici que Jaccottet accorde à tout discours une dimension foncièrement argumentative mais exprime son idéal d'un discours qui toucherait son auditeur en renonçant à cette visée perlocutoire. Il y a là bien sûr une illusion, car le lecteur ne pourra adhérer au texte que s'il lui attribue une visée, et, en l'occurrence, s'il se satisfait de cette mise en place de rapports et en perçoit la subtilité. De tels poèmes affichent des partis pris qui font sens dans l'histoire des esthétiques, mais leur énonciateur assume en les écrivant deux risques qui sont inhérents à son positionnement :

- au niveau du dit, argumenter sans recourir à des marqueurs explicites de subjectivité qui orienteraient vers une vision euphorique ou dysphorique de la réalité décrite expose au risque de bloquer la relation d'empathie avec le lecteur : celui-ci ne sait pas quelle leçon tirer de la description, il la perçoit comme gratuite et peu pertinente.

- au niveau du dire, s'interdire toute amplification, tout brillant, expose au reproche d'une écriture plate, sans intérêt.

On le voit bien ici, si argumenter, c'est se positionner dans un débat, l'effacement des marques argumentatives explicites, et, au-delà, des subjectivèmes transparents du locus amoenus, n'est qu'une autre façon d'argumenter et de tels choix d'écriture, délestant le texte de toute intentionnalité apparente, peuvent diviser les lecteurs à la fois sur le plan de l'engagement éthique ${ }^{23}$ et sur celui de l'appréciation esthétique. Des problèmes en partie similaires sont posés par les textes allégoriques qui sont plus fréquents qu'on ne croit dans la poésie contemporaine et qui, eux aussi, permettent d'argumenter par la schématisation mais avec de plus un rapport d'analogie implicite entre le thème et le phore $^{24}$.

\section{Conclusion}

33 J'ai voulu dans cet article distinguer assez nettement deux modes d'argumentation en poésie qui appellent à mon sens deux attitudes de lecture distinctes. Je me suis tout d'abord intéressée à des textes articulés sur des conflits de points de vue qui affichent leur dialogisme en recourant à des marqueurs bien étudiés par la théorie de l'argumentation dans la langue et par la linguistique énonciative: connecteurs, négations, discours rapportés. J'ai envisagé ensuite un cas particulier de rapport au discours de l'autre, interne au champ littéraire, qui a suscité des genres spécifiques, le travestissement et le pastiche. Enfin j'ai examiné des textes qui se présentent comme fondamentalement descriptifs, et qui me semblent relever clairement de ce que Rabatel (2004, § 47-49) appelle l'argumentation indirecte, plus particulièrement dans la deuxième formulation qu'il en donne :

Hypothèse $n^{\circ} 2$ :

1a : Il y a bien un énoncé E2 ; mais l'énoncé E1 n'est pas explicite [...];

2a: Il y a bien une réponse; mais celle-ci prend la forme d'un énoncé descriptif, en l'absence de question explicite [...]

3 : Il n'y a pas d'argument explicitement proféré par un locuteur ; mais les états $d u$ monde dénotés (valeur descriptive) se doublent d'une valeur argumentative (interprétative) à valeur d'argument, sur le mode des évidences perceptuelles ou conceptuelles (italiques d'A. Rabatel). 
Dans le cas des travestissements et pastiches, l'énoncé E1 est encore présent allusivement et peut, plus ou moins facilement, être retrouvé mais il s'agit d'un texte entier, d'une œuvre, voire d'une tradition discursive. On peut également remonter à un texte $\mathrm{E} 1$ dans le cas des textes épidictiques relevant de l'effacement énonciatif, qui prennent le contrepied des textes épidictiques canoniques. Mais, au-delà de leurs différences, les poèmes des troisième et quatrième parties partagent trois caractéristiques essentielles :

- c'est leur forme même qui se fait argumentation, soit par rapport à un certain état du champ littéraire, soit en suggérant au lecteur un sens oblique ${ }^{25}$ (ce que Ponge appelait une « leçon») : cette forme régit à la fois le contenu (thèmes, référents) et l'expression (lexique, syntaxe, rythme) - on passe ainsi d'une argumentation dans le poème (partie 2) à une argumentation $d u$ poème (parties 3 et 4$)$;

- leur dimension argumentative s'exerce dans deux directions et apporte une réponse à deux questions à la fois: "quelles sont les valeurs qui doivent guider notre être au monde? " et "comment en parler adéquatement?». Ces questions très générales peuvent bien sûr être spécifiées en fonction des situations socio-discursives qui déterminent la production du texte: «comment écrire quand on est frappé par un deuil ?", " comment écrire sur l'humain sans tomber dans la psychologie et l'effusion des sentiments ? ", « comment exprimer notre rapport au cosmos? », etc.

- l'implicitation de l'argumentation a pour conséquence que le lecteur peut passer à côté du dialogisme de ces poèmes (ignorer par exemple la façon dont l'auteur, par ce texte, revendique une certaine position dans le champ littéraire), mais aussi de leur dimension éthique, point sur lequel je m'attarderai un peu pour finir.

Le passage mal compris de Jakobson (1963) sur l'autotélisme de la fonction poétique ainsi que l'hégémonie du structuralisme - qui, lui-même, réagissait au tout historique de la critique traditionnelle - ont conduit pendant un certain nombre d'années la majorité des critiques à se désintéresser de la dimension éthique de la poésie et à privilégier dans l'étude des textes la façon dont ils répondaient à la question " comment écrire?». Pourtant les poètes ne cessaient d'affirmer qu'ils écrivaient "pour mieux vivre» (réponse de Saint-John Perse à une enquête). S'intéresser à la valeur argumentative de ces textes en analysant l'intrication entre leur dimension éthique et leur dimension esthétique me parait faire justice à la fois à cette affirmation des auteurs relayée par leurs lecteurs ${ }^{26}$ et aux caractéristiques intrinsèques de ces textes qui, par l'usage qu'ils font du langage, non seulement décrivent le monde mais cherchent à nous le faire éprouver, parfois sur le mode de l'évidence, d'autres fois, sur le mode du conflit cognitif et de la mise en débat. Cultivant tantôt l'homonoia, tantôt la dissidence, parfois l'intranquillité, et d'autres fois encore l'absorption du moi dans le monde, les poèmes sont leçons de vie autant que de langage ${ }^{27}$. Cela devrait nous inciter, dans notre enseignement, à développer chez les lecteurs novices une capacité de lecture où la jouissance esthétique ne soit pas séparée d'une prise de conscience du positionnement socio-historique du poète mais aussi des enjeux éthiques de ses textes. 


\section{BIBLIOGRAPHIE}

\section{Cuvres citées ou étudiées}

Fourest, Georges. 1913. La négresse blonde (Paris : Georges Crès et Cie)

Gaspar, Lorand. 2004 [2001]. Patmos et autres poèmes (Paris : Poésie /Gallimard)

Grosjean, Jean. 1996. Nathanaël (Paris : Gallimard)

Jaccottet, Philippe. 1988. La Promenade sous les arbres (Lausanne : La Bibliothèque des Arts)

Jaccottet, Philippe. 1994 [1977]. À la lumière d'hiver précédé de Leçons et de Chants d'en bas, suivi de Pensées sous les nuages (Paris : Poésie /Gallimard)

Michaux, Henri. 2001 [1949]. La vie dans les plis dans Euvres complètes, vol. 2, Bibl. de la Pléiade

(Paris : Gallimard)

Michot, Jacques-Henri. 1998. Un ABC de la barbarie (Marseille : Al Dante)

Proust Marcel. 1971 [1919]. Contre Sainte-Beuve précédé de Pastiches et mélanges et suivi de Essais et articles. Bibl. de la Pléiade (Paris : Gallimard)

Reboux, Paul et Müller, Charles. 1913. À la manière de... (Paris : Grasset)

Rimbaud, Arthur. 1999. CEuvres complètes, Brunel Pierre (éd.), La Pochothèque (Paris : Le Livre de Poche)

\section{Références}

Adam, Jean-Michel \& André Petitjean. 1989. Le texte descriptif (Paris : Nathan)

Adam, Jean-Michel. 1990. Éléments de linguistique textuelle (Bruxelles : Mardaga)

Amossy, Ruth. 2012 [2000]. L'argumentation dans le discours (Paris : Colin)

Aron, Paul. 2002. Article " pastiche », Aron Paul, Denis Saint-Jacques \& Alain Viala (éds).

Dictionnaire du littéraire (Paris : PUF)

Aron, Paul. 2009. « Le pastiche comme objet d'étude littéraire. Quelques réflexions sur l'histoire du genre ", Modèles linguistiques 60, en ligne URL : http://ml.revues.org/205

Bakhtine, Mikhaïl. 1978. Esthétique et théorie du roman, trad. par Daria Olivier (Paris : Gallimard)

Barthes, Roland. 1968. « L'effet de réel », Communications 11, 84-89

Bourdieu, Pierre. 1992. Les règles de l'art : genèse et structure du champ littéraire (Paris : le Seuil)

Bres, Jacques, Aleksandra Nowakowska \& Jean-Marc Sarale. 2016. « Anticipative interlocutive dialogism: sequential patterns and linguistic markers in French », Journal of pragmatics, 96, 80-95

Camusso, Julie. 2009. «Les pastiches-Baudelaire : un florilège vénéneux », Modèles linguistiques 60, en ligne URL : http://ml.revues.org/218

Dominicy, Marc. 2009. «La théorie des actes de langage et la poésie », L’Information Grammaticale 121, « Linguistique du texte poétique », 40-45

Dominicy, Marc. 2011. Poétique de l'évocation. (Paris : Classiques Garnier) 
Doury, Marianne. 2010. " "Un cimetière et des avions" : argumentation et valeurs dans le courrier des lecteurs d'un journal local », Argumentation et analyse de discours 5, « La lettre, laboratoire de valeurs? » en ligne : URL : http://aad.revues.org/1003

Franken, Nathalie \& Marc Dominicy. 2001. « Epidictique et discours expressif », Dominicy, Marc \& Madeleine Frédéric. La mise en scène des valeurs (Lausanne : Delachaux \& Niestlé), 79-106

Genette, Gérard. 1982. Palimpsestes (Paris : Seuil)

Grize, Jean-Blaise. 1997. Logique naturelle et communications (Paris : PUF)

Hamon, Philippe. 1994 [1981]. Du descriptif (Paris : Hachette Supérieur)

Jakobson, Roman. 1963. Essais de linguistique générale, tome 1, trad. N. Ruwet (Paris : Minuit)

Kissine, Mikhail. 2013. From Utterances to Speech Acts (Cambridge: Cambridge University Press)

Koren, Roselyne. 2004. «Argumentation, enjeux et pratique de l'« engagement neutre » : le cas de l'écriture de presse ", Semen 17 " Argumentation et prise de position : pratiques discursives », en ligne : URL : http://semen.revues.org/230

Lebel, Jean. 2002. Article « Description » Aron Paul, Denis Saint-Jacques \& Alain Viala (éds). Dictionnaire du littéraire (Paris : PUF)

Maingueneau, Dominique. 2004. Le Discours Littéraire. Paratopie et Scène d'Enonciation (Paris : Colin)

Maulpoix, Jean-Michel. 2009. Pour un lyrisme critique (Paris : Corti)

Michaux, Christine \& Marc Dominicy. 2001. « Le jeu réciproque du cognitif et de l'émotif dans le genre épidictique ", Dominicy, Marc \& Madeleine Frédéric (éds). La mise en scène des valeurs (Lausanne : Delachaux \& Niestlé), 135-165

Micheli, Raphaël. 2012. « Les visées de l'argumentation et leurs corrélats langagiers : une approche discursive ", Argumentation et analyse du discours 9, «L'analyse du discours entre critique et argumentation », en ligne URL : http://aad.revues.org/1406

Mellet, Sylvie \& Michèle Monte. 2008. « Néanmoins et toutefois » Mellet, Sylvie (éd.). Concession et dialogisme. Les connecteurs concessifs à l'épreuve des corpus (Berne : P. Lang), 55-85

Monte, Michèle. 2002, Mesures et passages. Une approche énonciative de l'œuvre poétique de Philippe Jaccottet (Paris : Champion)

Monte, Michèle. 2003. « Essai de définition d'une énonciation lyrique. L'exemple de Philippe Jaccottet », Poétique 134, 159-181

Monte, Michèle. 2006. « Runes de Jean Grosjean et La grande neige d'Yves Bonnefoy : de l'étrangeté pragmatique à la lecture allégorique », Brophy, Michael \& Mary Gallagher (éds). Sens et présence du sujet poétique. La poésie de la France et du monde francophone depuis 1980 (Amsterdam/ New York : Rodopi), 227-241

Monte, Michèle. 2008. «Le jeu des points de vue dans l'oxymore : polémique ou reformulation? », Langue française 160, « Figures et point de vue », 37-53

Monte, Michèle. 2010, « Scénographie mouvante et hétérogénéité des points de vue dans L'Âge de l'Humanité d'André Salmon », Monte Michèle (éd.). André Salmon, poète de l'Art vivant, (Faculté de Lettres de l’Université de Toulon, coll. « Var et poésie ») 193-214

Monte, Michèle. 2011a. «Effacement énonciatif et pratique citationnelle dans Égée de Lorand Gaspar : une cohérence globale », Bougault, Laurence \& Judith Wulf (éds), Lorand Gaspar et la langue (Rennes : Éd. Styl-m), 105-150 
Monte, Michèle. 2011b, « Pastiches baudelairiens, éthos et style », Gardes Tamine, Joëlle \& Georges Molinié (éds). Style et création littéraire (Paris : Champion), 25-39

Monte, Michèle. 2012. « Pour une autonomie relative des niveaux sémantique, énonciatif et iconique des textes poétiques ", Actes du $3^{e}$ CMLF, (Paris : EDP Sciences), 1199-1213, en ligne DOI: http://dx.doi.org/10.1051/shsconf/20120100044

Monte, Michèle. 2016. « De l'éthos, du style et du point de vue en poésie », Colas-Blaise, Marion, Laurent Perrin \& Gian Maria Tore (éds). L'énonciation aujourd'hui, un concept clé des sciences du langage (Limoges : Lambert-Lucas), 179-200

Monte, Michèle. À paraitre. «Les relations entre énonciateur textuel, locuteurs et personnages dans quelques scénographies poétiques ", Biglari, Amir \& Nathalie Watteyne (éds). L'énonciation lyrique (Paris : Classiques Garnier)

Monte, Michèle \& André Bellatorre. 2008. Le printemps du temps. Poétiques croisées de Francis Ponge et Philippe Jaccottet (Aix-en-Provence : PUP)

Plantin, Christian. 2016. Dictionnaire de l'argumentation (Lyon : ENS éditions)

Rabatel, Alain. 2004. « Effacement énonciatif et effets argumentatifs indirects dans l'incipit du "Mort qu'il faut" de Semprun », Semen17 « Argumentation et prise de position : pratiques discursives », en ligne URL : http://semen.revues.org/2334

Rabatel, Alain. 2008. «Figures et points de vue en confrontation », Langue française 160, « Figures et point de vue », 3-19

Rabatel, Alain. 2009. « Prise en charge et imputation, ou la prise en charge à responsabilité limitée... », Langue française 162 « La notion de prise en charge en linguistique », 71-87

Rabatel Alain. 2017. Pour une lecture linguistique et critique des médias. Empathie, éthique, point(s) de vue (Limoges : Lambert-Lucas)

Rodriguez, Antonio. 2003. Le pacte lyrique. Configuration discursive et interaction affective (Bruxelles : Mardaga)

\section{NOTES}

1. Je laisserai de côté ici la poésie épique et la poésie didactique qui me semblent relever de régimes énonciatifs partiellement distincts. Sur la question du lyrique conçu comme un pacte de lecture, on pourra lire Rodriguez (2003) et Monte (à paraitre).

2. «[L]a simple transmission d'un point de vue sur les choses, qui n'entend pas expressément modifier les positions de l'allocutaire, ne se confond pas avec une entreprise de persuasion soutenue par une intention consciente et offrant des stratégies programmées à cet effet » (2012 : 44).

3. L'étude de la répartition des connecteurs concessifs par genre que j'avais menée avec Sylvie Mellet avait bien montré une sous-représentation de ces connecteurs dans la poésie (Mellet et Monte 2008 : 58) mais avec des exceptions chez certains auteurs.

4. Le découpage en paragraphes est déroutant: on attendrait que «les lances...corps » qui joue argumentativement le même rôle que « Ils ont détruit son "un" soit isolé par un alinéa. Michaux suggère sans doute par là que ce qui fait la faiblesse du Meidosem est aussi ce qui fait sa force et peut donc figurer aussi bien avant qu'après « mais ».

5. Notons que cette aspiration n'est pas exprimée dans un discours rapporté attribué au Meidosem mais inférée de l'interrogation rhétorique «Quel étranglé ne parle un jour de se 
libérer? » où L1/E1 prend le lecteur à témoin d'une vantardise commune à toute une classe dont font partie les Meidosems.

6. On trouvera deux analyses détaillées de ces stratégies citationnelles à l'échelle d'une œuvre entière dans Monte (2010 et 2011a).

7. Le choix lexical pour désigner les femmes qui s'occupent du mourant épouse le PDV qui voit dans la mort une « autre naissance ».

8. J’appelle ainsi des groupes de vers en poésie non métrique.

9. Genette fait remarquer à juste titre que, dans l'usage courant, et même dans les dictionnaires, ces trois catégories sont fréquemment confondues (1982: 37), voir également l'article «pastiche » d'Aron (2002).

10. Genette évoque ces poèmes aux p. 89-91 de Palimpsestes.

11. Écrit en 1870 et adressé à Demeny, il est publié pour la première fois en 1891 dans Reliquaire (voir Rimbaud 1999 : 780).

12. Les parallélismes entre le vers 5 et le vers 8 montrent sans la dire une correspondance entre la culotte et les étoiles, le trou et le frou-frou. Le frou-frou étant associé aux vêtements féminins, les connotations sexuelles abondent.

13. Sur ces cas de dissociation, voir Monte (à paraître).

14. La première moitié du $20^{\mathrm{e}}$ siècle a vu fleurir des recueils de pastiches où, sur un même sujet, l'auteur ou les auteurs imitai[en]t successivement des écrivains différents. Proust (1971 [1919]) le fait sur le mode sérieux dans L'Affaire Lemoine, et Reboux et Müller (1913) de façon plaisante dans À la manière de...

15. On trouvera, dans Camusso (2009), une première analyse de ce texte ainsi que de dix-sept autres pastiches de Baudelaire, et dans Monte (2011b) une étude stylistique de ces mêmes textes. 16. Sur ces deux modes d'intégration de l'énoncé ou PDV autre, auxquels il faut ajouter la prolepse qui commence par donner la parole à l'opposant, voir Bres et al, art. cité. Notons que la praxématique préfère parler d'image d'énoncé que de point de vue, terme que je reprends à Rabatel.

17. Il est significatif du parti pris de Plantin que l'entrée «épidictique » soit absente de son dictionnaire.

18. Dans les textes explicitement argumentatifs, la schématisation contribue à asseoir la thèse défendue. Dans les textes épidictiques, c'est à elle seule qu'incombe cette tâche.

19. Dans leur théorie des actes de langage $(2009,2013)$, ces énoncés sont décrits comme des actes locutoires exprimant une croyance mais sont dépourvus de valeur illocutoire, L1/E1 ne donnant pas de raison à l'allocutaire pour croire ce que l'acte locutoire a formulé. Pour Dominicy, cette analyse convient mieux aux textes poétiques qu'une analyse rhétorique « moniste » qui voit dans l'énoncé poétique, au moins au niveau de L1/E1, un énoncé comme les autres. J'assume, quant à moi, jusqu'à présent cette position moniste (voir Dominicy 2011 et Monte 2012) tout en reconnaissant que la théorie de l'évocation, qui, elle, postule l'hétérogénéité mutuelle des organisations linguistique et poétique, rend beaucoup mieux compte du rôle du vers dans la poésie.

20. Le seul posé des énoncés exclamatifs est l'intensité.

21. On a là affaire typiquement à des concepts inanalysés que Dominicy (2011) estime essentiels dans le mécanisme de l'évocation poétique.

22. La dimension problématisante de cette attitude est difficile à percevoir en dehors d'une bonne connaissance de l'histoire de la poésie, d'où la perplexité de certains lecteurs qui m'a amenée à parler d'« objets pragmatiquement non identifiés » pour ce type de poème (Monte 2006).

23. J'entends ici cet adjectif comme un dérivé d'ethos : tout texte est porté par un ton rattachable à un ethos et, dans le cas du texte littéraire, cherche à susciter en retour une incorporation de cet ethos par le lecteur (Maingueneau 2004). 
24. Je réserve leur étude à un autre article.

25. Ce sens oblique devient double sens dans l'allégorie, qui est une sorte d'analogie.

26. Y compris dans des situations extrêmes comme celles que durent affronter Primo Levi, Jorge Semprun ou Varlam Chalamov, qui, tous, évoquent la récitation de poèmes dans les camps.

27. Monte et Bellatorre (2008) s'intéressent précisément à cette articulation dans le cas de Ponge et Jaccottet.

\section{RÉSUMÉS}

Cet article défend l'idée que les poèmes possèdent une argumentativité intrinsèque, mais graduelle, allant de formes très explicites (mise en scène de conflits de points de vue, dialogisme interlocutif et interdiscursif) à des formes implicites, reposant sur les schématisations. La démonstration s'appuie sur l'étude de poèmes variés (Rimbaud, Jaccottet, Michaux, Grosjean, Gaspar) et s'attarde tout particulièrement, d'une part sur l'argumentation qui porte sur la littérature elle-même par le recours au travestissement et au pastiche, d'autre part sur les textes épidictiques, qu'ils recherchent l'expressivité ou reposent au contraire sur l'effacement énonciatif. L'imbrication qu'opèrent les poèmes entre choix formels et contenus thématiques conduit à affirmer que l'éthique et l'esthétique y sont étroitement liés, ce que l'analyse devrait prendre en compte.

This paper argues that poems are intrinsically but gradually argumentative. It describes both explicit argumentative forms (conflicting points of view, interlocutive and interdiscursive dialogism) and implicit ones, through what Grize called schematizations. Focusing on the study of several poems (by Rimbaud, Jaccottet, Michaux, Grosjean, Gaspar), it pays a particular attention to argumentation dealing with literature itself (through burlesque and pastiche), and to epidictic texts seeking for expressivity or obliterating their speaker. The close connection between formal choices and thematic contents in poetry shows that it tightly links ethics with aesthetics, a feature that the analyst should take into account.

argumentation, dialogism in poetry, epidictic, ethics, hypertextual genres, implicit, reader, $20^{\text {th }}$ century French poetry

\section{INDEX}

Mots-clés : argumentativité, dialogisme du poème, épidictique, éthique, genres hypertextuels, implicite, lecteur, poésie française du 20e siècle

\section{AUTEUR}

\section{MICHĖLE MONTE}

Université de Toulon, Babel EA 2649 